\title{
Comportamentos observados em tenistas com diferentes níveis de desempenho máximo em competições amadoras
}

\section{Observed behaviors in tennis players with different levels of maximum performance in amateur competition}

\author{
Edirley Ronaldo Costa \\ Universidade Estácio de Sá - UNESA - Rio de Janeiro - Brasil \\ ronaldo.tenis@hotmail.com \\ Roberto Tierling Klering \\ Universidade Federal do Rio Grande do Sul - UFRGS - Porto Alegre - Brasil \\ robertoklering@gmail.com \\ Caio Corrêa Cortela \\ Universidade Federal do Rio Grande do Sul - UFRGS - Porto Alegre - Brasil \\ caio.tenis@yahoo.com.br \\ Gabriel Henrique Treter Gonçalves \\ Universidade Federal do Rio Grande do Sul - UFRGS - Porto Alegre - Brasil \\ gabrielhtg@gmail.com \\ Carlos Adelar Abaide Balbinotti \\ Universidade Federal do Rio Grande do Sul - UFRGS - Porto Alegre - Brasil \\ carlos.balbinotti@ufrgs.br
}

\section{Resumo}

No Brasil, o tênis amador representa uma relevante parcela do mercado de trabalho de professores e treinadores de tênis. O objetivo desse estudo é descrever e comparar as médias de: a) aproveitamento de primeiro saque; b) pontos ganhos jogados com o primeiro saque; c) duplas-faltas; d) erros forçados; e) erros não-forçados; f) número de trocas de bola; e g) tempo de duração das trocas de bola, entre três categorias masculinas: $1^{a} \mathrm{~A} ; 3^{a} \mathrm{~A}$; e $5^{\mathrm{a}} \mathrm{A}$, da Federação Paranaense de Tênis. Foram coletados dados de 15 jogos, cinco de cada categoria analisada. Os dados foram analisados por meio do software SPSS 15.0. Os resultados indicaram: a) maior aproveitamento de primeiro saque $(p<0,05)$ da categoria $1^{a} A$ em relação à categoria $\left.5^{a} \stackrel{A}{A} ; b\right)$ número inferior de duplas-faltas e erros forçados da categoria $1^{\mathfrak{a}} \mathrm{A}$ em relação à $3^{\mathrm{a}} \mathrm{A}$ e $5^{\mathrm{a}} \mathrm{A} ; \mathrm{c}$ ) maior número de trocas de bola para a $1^{\mathrm{a}} \mathrm{A}$ nas situações de 6-10 e acima de 10 trocas em relação às classes $3^{\mathrm{a}} \mathrm{A} \mathrm{e}$ $5^{\mathrm{a}} \mathrm{A}$. Observa-se que, para as categorias $3^{\mathrm{a}} \mathrm{A}$ e $5^{\mathrm{a}} \mathrm{A}$ atingirem a $1^{\mathrm{a}} \mathrm{A}$, é preciso uma intensificação no treinamento de saque e dos golpes defensivos/neutralizantes, diminuindo o número de duplas-faltas e de erros forçados.

Palavras-chave: Tênis, Análise de desempenho, Comparação. 


\begin{abstract}
In Brazil, amateur tennis represents a relevant share of the labor market for tennis teachers and coaches. The objective of this study is to describe and compare means of: a) \% first serve in; b) first serve points won; c) double-faults; d) forced errors; e) unforced errors; f) rally length (number of strokes); and g) rally length (time), among three men categories: $1^{\text {st }} A ; 3^{\text {rd }} A$; and $5^{\text {th }} A$, of the Paraná Tennis Federation. Data were collected from 15 matches; five from each analyzed category. Data were analyzed using the software SPSS 15.0. Results indicated: a) more first serves in $(p<0,05)$ for $1^{\text {st }} A$ category when compared to $5^{\text {th }} A$ category; b) less double-faults and forced errors for $1^{\text {st }} A$ category when compared to $3^{\text {rd }} \mathrm{A}$ and $5^{\text {th }} \mathrm{A} ; \mathrm{c}$ ) higher number of strokes during rallies for $1^{\text {st }} \mathrm{A}$ category in situations of 6-10 strokes and more than 10 strokes when compared to $3^{\text {rd }} A$ and $5^{\text {th }} A$ categories. It is observed that, for categories $3^{\text {rd }} A$ and $5^{\text {th }} A$, to achieve $1^{\text {st }} A$ category level, it is necessary practice intensification regarding serve and defensive/neutralizing strokes, reducing the number of double-faults and forced errors.
\end{abstract}

Keywords: Tennis, Performance analysis, Comparison.

\title{
1. Introdução
}

O desempenho nas competições é influenciado por muitas variáveis, dentre elas, destacam-se as físicas, psíquicas, técnicas e táticas. Por isso, a preparação para uma competição deve ser abrangente, onde é necessário entender as respostas e características do jogador no momento em que ele compete. Nesse sentido, a área de estudo que contempla a análise de desempenho de jogo tem se especializado cada vez mais, coletando dados mais detalhados sobre o esporte. Não obstante, o avanço da tecnologia também torna possível a análise de competições amadores, antes só possível com grande investimento e, com isso, limitada às competições profissionais.

A coleta de dados no esporte amador permite a comparação de desempenho entre atletas de mesmo e de diferentes níveis, encontrando, com isso, caminhos e orientações para treinadores planejarem suas sessões de treinamento. De acordo com Bompa (2002), esses caminhos e orientações são denominados de modelação esportiva, e, segundo o autor, é um dos princípios mais importantes para o treinamento esportivo.

Nesse contexto, o presente estudo tem como objetivo descrever e comparar as médias de: a) aproveitamento de primeiro saque; b) pontos ganhos jogados com 0 primeiro saque; c) duplas-faltas; e) erros forçados; f) erros não-forçados; g) número de trocas de bola; e h) tempo de duração das trocas de bola, entre três categorias masculinas: $1^{\underline{a}}$ Classe A; $3^{\underline{a}}$ Classe A; e 5 ${ }^{\underline{a}}$ Classe A, da Federação Paranaense de Tênis.

\section{Materiais e métodos}

A amostra deste estudo foi composta por 15 jogos de três categorias competitivas amadoras da Federação Paranaense de Tênis (FPT). Cada categoria teve cinco jogos analisados. Esses jogos faziam parte do calendário de Torneios da FPT, e foram realizados nas cidades de Londrina, Maringá, Apucarana e Arapongas. As categorias analisadas foram 1ำ (avançados), 3 A (intermediários) e $5^{\circ}$ A (iniciantes). Foram analisadas as semifinais e sorteados três jogos de quartas de final para integrarem a amostra. Fizeram parte da pesquisa 25 jogadores do sexo masculino, onde os jogadores da 5A tinham uma média de idade de 16,05 anos e 4,75 anos de prática; os jogadores da 3ํA tinham idade média de 19,38 anos e 9,63 anos de prática; e os jogadores da 1ํA tinham uma média de idade de 22,38 anos e 11,63 anos de prática.

A coleta de dados foi feita no local de cada torneio, com a permissão do árbitro geral do torneio e de cada atleta referente ao jogo sorteado. A coleta de dados foi 
realizada com o software Tennis Math, sistema operacional Android. Cada jogo foi acompanhado in loco, desde o seu início até o seu fim. As estatísticas coletadas foram: acerto de primeiro saque, pontos vencidos com o primeiro saque, duplas-faltas, erros forçados, erros não-forçados, trocas de bola por rali e tempo de duração dos ralis.

Em relação às análises, foi utilizado o software SPSS 15.0 para rodá-las. Assim, foram realizadas as estatísticas descritivas de tendência central (média, desvio padrão, média aparada a 5\%, mediana, e valores mínimo e máximo). Após, para as análises de comparação entre as categorias do estudo $\left(1^{\circ} \mathrm{A}, 3^{\circ} \mathrm{A}\right.$ e $\left.5^{\circ} \mathrm{A}\right)$, utilizou-se a análise de variância e o testes não-paramétricos de Mann-Whitney e Kruskal-Wallis, todos a um nível de $5 \%$ de significância.

\section{Resultados e discussão}

A Tabela 1 apresenta os resultados das análises dos jogos dos jogos observados. Foi encontrada uma diferença estatisticamente significativa $(p<0,05)$ entre as categorias $1^{\circ} \mathrm{A}$ e 5 $\mathrm{A}$ no que diz respeito ao aproveitamento de $1^{\circ}$ saque. A experiência técnica e de torneios, de maneira geral, tendem a fazer com que os jogadores da $1^{\circ} \mathrm{A}$ tenham um melhor aproveitamento no $1^{\circ}$ saque. Isso pode revelar uma maior necessidade de simulação de jogos que estimulem mais a prática do saque no sentido de aprimorar a técnica dos jogadores da 5ํㅗ. Essas observações também servem à diferença estatisticamente significativa $(p<0,05)$ encontrada no número de Duplas-Faltas da categoria $1^{\circ} \mathrm{A}$ para as categorias $3^{\circ} \mathrm{A}$ e $5^{\circ} \mathrm{A}$.

Tabela 1: Estatística descritiva: tendência central e dispersão

\begin{tabular}{|c|c|c|c|c|c|c|c|c|}
\hline \multirow[b]{3}{*}{ Categorias } & \multirow{3}{*}{$\begin{array}{l}\text { Dimensão } \\
\text { analisada }\end{array}$} & \multirow[b]{3}{*}{$\mathbf{N}$} & \multicolumn{6}{|c|}{ Tendência Central e Dispersão } \\
\hline & & & \multirow[b]{2}{*}{$\bar{X}$} & \multirow[b]{2}{*}{ DP } & \multirow[b]{2}{*}{$5 \%$} & \multirow[b]{2}{*}{ Mediana } & \multicolumn{2}{|c|}{ Amplitude } \\
\hline & & & & & & & Mínima & Máxima \\
\hline & \%Saque & 10 & 70,40 & 13,81 & 71,28 & 73,50 & 37 & 88 \\
\hline & $\%$ Pts. 1 @Saque & 10 & 60,60 & 13,85 & 60,72 & 63,50 & 37 & 82 \\
\hline \multirow[t]{5}{*}{ 1․ Classe A } & Duplas -faltas & 10 & 1,5 & 1,65 & 1,38 & 1,5 & 0 & 5 \\
\hline & Erros Forçados & 10 & 13,20 & 2,48 & 13,22 & 13,50 & 9 & 17 \\
\hline & Erros Ñ Forçados & 10 & 24,20 & 9,16 & 24,27 & 25 & 11 & 36 \\
\hline & \%Saque & 10 & 65,10 & 9,17 & 65,06 & 65 & 53 & 78 \\
\hline & $\%$ Pts. $1^{\circ}$ Saque & 10 & 58,40 & 15,62 & 59,50 & 64 & 23 & 74 \\
\hline \multirow[t]{5}{*}{ 3a Classe A } & Duplas -faltas & 10 & 6,1 & 4,7 & 5,72 & 4,5 & 2 & 17 \\
\hline & Erros Forçados & 10 & 17,60 & 6,58 & 17,77 & 18 & 5 & 27 \\
\hline & Erros Ñ Forçados & 10 & 30 & 10,10 & 29,88 & 29 & 13 & 49 \\
\hline & \%Saque & 10 & 51,70 & 13,50 & 51,17 & 50,50 & 35 & 78 \\
\hline & $\%$ Pts. $1^{\circ}$ Saque & 10 & 55,40 & 13,38 & 56,67 & 59 & 21 & 67 \\
\hline \multirow[t]{3}{*}{ 5 Classe A } & Duplas -faltas & 10 & 5,6 & 2,45 & 5,55 & 5 & 3 & 9 \\
\hline & Erros Forçados & 10 & 23 & 7,45 & 23,22 & 24,50 & 8 & 34 \\
\hline & Erros Ñ Forçados & 10 & 30,10 & 9,26 & 30,44 & 33,50 & 14 & 40 \\
\hline
\end{tabular}

Fonte: Pesquisa de campo (2016)

Os pontos ganhos com o primeiro saque e os erros não-forçados não apresentaram diferenças significativas entre as três categorias analisadas. Para os pontos ganhos com o primeiro saque, Carvalho, Aburachid e Greco (2007) destacam que há vantagens do sacador em relação ao devolvedor; sendo o "ace", o erro de devolução e a vantagem no posicionamento de quadra fatores decisivos para o sacador lograr, geralmente, mais êxitos nos pontos durante o jogo. Uma vez que o sacador executa 0 
saque no centro da quadra e o devolvedor aguarda o saque na lateral da quadra, quando a devolução retorna à quadra, o sacador cria um espaço vazio para tirar vantagem tática. Nesse sentido, ter o $1^{\circ}$ saque eficiente interfere diretamente na consequência da jogada; isso serve tanto para jogadores avançados como para jogadores intermediários e/ou iniciantes.

Os Erros Não-Forçados são erros originados das ações do próprio jogador, ou seja, não é uma consequência direta da ação de seu oponente (FERNANDES et al., 2013). Enquanto os erros forçados são erros onde o jogador não consegue responder a bola dentro da quadra adversária de forma efetiva, em decorrência de haver recebido um golpe potente e/ou preciso, que dificultou a sua ação.

Para os Erros Forçados, os resultados mostram diferenças significativas $(p<0,05)$ entre a categoria $1^{\circ} \mathrm{A}$ e as categorias $3^{\circ} \mathrm{A}$ e $5^{\circ} \mathrm{A}$. Assim, o que se observa é que os jogadores da 1ำ apresentam um número menor de erros forçados durante a competição. Para diminuir os erros forçados, os jogadores devem ser capazes de defender ou de neutralizar os ataques do oponente. Para tanto, algumas qualidades como, por exemplo, a maturidade tática nos ralis, reposicionamento em quadra consistente, habilidades de antecipação evoluídas e tomada de decisões mais rápidas são fundamentais. Embora o estudo não tenha avaliado essas variáveis, elas podem ser apontadas como prováveis causas da diferença entre as categorias analisadas. Contudo, novos estudos precisam ser feitos para que essa suspeita seja confirmada.

Os Erros Não-Forçados não apresentaram diferenças significativas $(p>0,05)$ entre as categorias; ainda que os valores absolutos sejam diferentes entre si. Para Balbinotti e Motta (2009, p. 159) 'tenistas com controle e regularidade de bola, mas sem potência, podem vencer jogos nos níveis iniciantes e intermediários, mas dificilmente vencerão no alto rendimento, pois nesta fase a potência representa uma qualidade determinante para a vitória". Os jogadores da 1스 arriscam mais, procurando ganhar mais pontos com bolas vencedoras. Seus erros não-forçados acontecem por intencionalidade ofensiva. Já os erros não-forçados da $3^{\circ}$ e $5^{\circ}$ classe tendem a ocorrer por deficiência técnica (falta de controle da força, organização ineficiente dos ângulos da raquete ao impactar a bola, pobre antecipação para recepção da bola e determinar linhas de corrida, entre outros).

Tabela 2: tempo de troca de bola e número de rebatidas por rali.

\begin{tabular}{|c|c|c|c|c|c|c|c|c|c|c|c|c|c|c|c|c|}
\hline \multirow{3}{*}{$\begin{array}{c}\text { Categoria } \\
\mathbf{s}\end{array}$} & \multicolumn{8}{|c|}{$\begin{array}{l}\text { Porcentagem de pontos disputados em função } \\
\text { do intervalo de trocas de bolas (rallys) }\end{array}$} & \multicolumn{8}{|c|}{ Tempo de duração dos rallys (s) } \\
\hline & \multicolumn{2}{|c|}{$0-1$} & \multicolumn{2}{|c|}{$2-5$} & \multicolumn{2}{|c|}{$6 .-10$} & \multicolumn{2}{|c|}{$10+$} & \multicolumn{2}{|c|}{$0-3 \mathrm{~s}$} & \multicolumn{2}{|c|}{$4-10 s$} & \multicolumn{2}{|c|}{$10-20 \mathrm{~s}$} & \multicolumn{2}{|c|}{$20 \mathrm{~s}$} \\
\hline & $\bar{X}$ & $D P$ & $\bar{X}$ & $D P$ & $\bar{X}$ & $D P$ & $\bar{X}$ & $D P$ & $\bar{X}$ & $D P$ & $\bar{X}$ & $D P$ & $\bar{X}$ & $D P$ & $\bar{X}$ & $\begin{array}{l}D \\
P\end{array}$ \\
\hline $1^{\mathrm{a}}$ Classe & 29,2 & 11,6 & 36,6 & 9,1 & 16,4 & 7,1 & 7,6 & 5,0 & 6,5 & 3,8 & 44,7 & 6,7 & 28,6 & 3,5 & 18,1 & \\
\hline $3^{\mathrm{a}}$ Classe & 41,8 & 8,1 & 40,5 & 8,9 & 11,7 & 1,5 & 1,0 & 1,6 & 3,8 & 2,4 & 49,4 & 6,2 & 29,4 & 7,0 & 9,1 & \\
\hline $5^{\mathrm{a}}$ Classe & 35,9 & 8,7 & 36,5 & 10,2 & 9,9 & 2,5 & 1,7 & 0,9 & 4,6 & 1,9 & 43,3 & 6,4 & 33,9 & 3,6 & 12,2 & , \\
\hline
\end{tabular}

Fonte: Pesquisa de campo (2016)

Não houve diferença estatisticamente significativa $(p>0,05)$ para os intervalos de trocas de bola entre $0-1$ e 2-5 entre as três categorias. No entanto, a partir dos intervalos de 6-10 rebatidas e acima de 10 rebatidas por ponto, houve diferença significativa ( $p<$ $0,05)$ entre a classe $1^{\circ} \mathrm{A}$ e as classes $3^{\circ} \mathrm{A}$ e $5^{\circ} \mathrm{A}$. Nesse sentindo, Cortela et al. (2011) encontrou números semelhantes comparando tenistas infantis no intervalo de troca de bolas, onde jogadores G1 (nível superior infantil) apresentaram $40 \%$ dos pontos jogados com trocas de bolas acima de 6 rebatidas e 14\% dos pontos tiveram mais de 9 rebatidas, 
enquanto a categoria G2 (nível inferior) observou frequências de $17 \%$ e $5 \%$ para os intervalos de trocas entre cinco e oito bolas e mais de nove trocas, respectivamente.

Assim como observado nos estudos de Cortela et al. (2011), verificou-se que a maioria dos pontos são finalizados entre a $2^{\circ}$ e $5^{\circ}$ troca de bola. Esta informação pode orientar os treinadores das três categorias, assim como categorias infantojuvenis, a projetar o treinamento levando em conta o que acontecerá na maioria dos pontos. Como se vê nos números apresentados, as categorias mais avançadas se diferem significativamente das demais categorias quando o rali passa da $5^{\circ}$ bola trocada, indicando uma maior consistência dos tenistas com melhor desempenho.

\section{Considerações finais}

O caminho dos tenistas da $3^{a} \mathrm{~A}$ e $5^{\mathrm{a}} \mathrm{A}$, para atingirem a $1^{\mathrm{a}} \mathrm{A}$, parece exigir uma intensificação no treinamento do saque e dos golpes defensivos/neutralizantes, diminuindo o número de duplas-faltas e de erros forçados. Além disso, verificou-se que a maioria dos pontos são finalizados até a quinta bola trocada; revelando, também, um curto tempo de duração dos ralis (quatro a dez segundos) nas três categorias analisadas. Assim, espera-se que essas informações possam orientar os treinadores no sentido de aprimorar os padrões de jogo a partir do saque e devolução de saque (tendo em vista que são iniciativas de jogadas que afetam diretamente o número de trocas de bola) e, também, o treinamento físico dos jogadores das três categorias, preparando-os para pontos mais curtos e de alta intensidade, adequando-se a realidade das competições.

\section{Referências}

BOMPA, T. Periodização: teoria e metodologia do treinamento. São Paulo: Phorte Editora, 2002.

BALBINOTTI. C.; MOTTA, M. M. A bola em jogo no tênis. O domínio das técnicas avançadas. In: BALBINOTTI, C. A. A. (Org.). O ensino do tênis, novas perspectiva de aprendizagem. Porto Alegre. Artmed, 2009, p. 141-162.

CARVALHO, F.; ABURACHID, L. M. C.; GRECO, P. J. Estudo dos efeitos de saque no tênis e diferentes pontuações e pisos no jogo. Revista Portuguesa de Ciências do Desporto, Porto, v, 7, n. 1, p. 36, 2007.

CORTELA, C. C.; NASCIMENTO R. J.; KIST C.; ROCHA D.N. A carga de treinamento e competição dos tenistas infantis paranaenses: um estudo comparativo sobre tenistas com diferentes níveis de desempenhos máximos. Revista Eletrônica - FAFIT-FACIC, Itararé, v. 02, n. 01, p. 1-13, jan./jun. 2011.

FERNANDES, G. B.; LOPES, M. C.; DIAS, J.; DINIZ, L. B. F. Relação dos erros não forçados com o resultado do jogo no torneio de tênis de Roland Garros 2013. Revista Mineira de Educação Física, Viçosa, edição especial, n. 9, p. 427-432, 2013. 